\title{
Polarization structure in the core of $1803+784$ : a signature of recollimation shocks?
}

\author{
T. V. Cawthorne \\ Jeremiah Horrocks Institute, University of Central Lancashire, Preston, Lancashire PR1 \\ 2HE, U.K. \\ and \\ S. G. Jorstad ${ }^{1}$ and A. P. Marscher \\ Institute for Astrophysical Research, Boston University, 725 Commonwealth Avenue, \\ Boston, MA 02215, U.S.A.
}

Received __; accepted _

\footnotetext{
${ }^{1}$ Astronomical Institute, St. Petersburg State University, Universitetsky Pr. 28, Petrodvorets, 198504, St. Petersburg, Russia.
} 


\begin{abstract}
The extragalactic radio source $1803+784$ is a BL Lac object that shows rapid variability and strong linear polarization. Very long baseline interferometry observations reveal that the core possesses a distinctive structure in polarized intensity with two maxima on axis and two minima symmetrically placed on either side. The approximately radial pattern of electric field polarization rods is reminiscent of the results obtained earlier by Cawthorne (2006) for conical shocks, but, individually, these do not reproduce the main features of the polarized intensity images. In numerical simulations and experiments, these shocks occur in pairs and help to stabilize jets as they adjust to changes in environment. Here, the polarization resulting from such structures is investigated using an approximate, analytical approach, by making some simple assumptions about the nature of the flow between two such shock waves. For fairly small viewing angles, it is found that a reasonable representation of the core polarization of $1803+784$ can be obtained. The similarity between the observed and model polarization supports the view that the core structure in $1803+784$ represents a recollimation shock, and that such shock waves may be responsible for the first disturbance and hence brightening of the quiescent flow in astrophysical jets.
\end{abstract}

Subject headings: galaxies: active-galaxies: jets individual $(1803+784)$ 


\section{Introduction}

When there is a mismatch between the pressure of a jet and the ambient pressure at the jet boundary, the result can be a series of pairs of shocks that take the form of cone-shaped surfaces. In each pair of shock surfaces, a collimating or forward-pointing cone is followed by a decollimating or backward-pointing cone. It is likely that these two shock fronts meet at a Mach disk, a small region over which the shock is planar and perpendicular to the axis.

Since astrophysical jets are emerging into regions of declining pressure and density, it seems probable that conical shocks are present and able to influence the observational properties of jets as they emerge from the active galactic nucleus. The effect of conical

shocks on astrophysical jets has been studied by a number of authors. Lind and Blandford (1985) considered their influence on the anisotropy of radiation from jets, demonstrating that the result of relativistic beaming can differ quite significantly from that predicted by the usual relativistic beaming factor. Falle and Wilson (1985) performed numerical simulations of the jet flow in M87, identifying the conical shocks in their simulations with the weak knots of radio emission between the nucleus and knot A. They found that, using the rate of decline of gas pressure predicted by X-ray observations, their simulation predicted the correct spacing of the knots.

Gómez et al. (1995, 1997) generated simulations of synchrotron emission from the output of hydrodynamic codes in which recollimation shocks were produced by initially over-pressured jets in external media with both constant and declining pressure. Gómez et al. (1997) and Agudo et al. (2001) used this approach to consider the interaction between moving shocks and standing conical shocks, concluding that such interaction displaces the stationary component downstream and gives rise to trailing components that follow the main outward-moving disturbance.

Cawthorne and Cobb (1990) and Cawthorne (2006) used a simple semi-dynamical 
model for a conical shock front to consider what the polarization signatures of such shocks might be. These models assumed that the upstream magnetic field is either disordered on scales small compared to the resolution of any relevant observations or a combination of a disordered field component and a component of field parallel to the axis, as is found in some astrophysical jets. The results were compared, for example, to polarization observations of the 0.7 arcsecond knot in 3C 380 (Papageorgiou et al. 2006), which shows a pattern reminiscent of some of the simulations from Cawthorne (2006). Observations that revealed a similar component in 3C 120 have been reported by Roca-Sogorb et al. (2010) and Agudo et al. (2012). In the latter paper it was argued that this too represents a conical shock. In $3 \mathrm{C} 120$, the case for the existence of a shock is strengthened by the large increase in surface brightness over approximately 7 years, which is interpreted as the the result of the formation of the shock.

It is sometimes proposed (e.g., Daly \& Marscher, 1988, Marscher et al. 2008) that the high-frequency core components of astrophysical jets may be regarded as the first in the series of collimating and decollimating shock pairs that result when the jet first becomes under-pressured. In this picture the jet is assumed to be essentially invisible during its initial stages because it is a steady flow and therefore 'cool'. Such shocks seem to be an essential feature of most AGN jets because, unless a jet evolves smoothly (which is not, apparently, the case for most jets, which contain bright and variable features) such shocks represent the only mechanism through which it can reach a form of equilibrium following changes in the pressure of the jet or of its environment. Indirect evidence for identification of the high-frequency radio cores with recollimation shocks comes from the work of Marscher et al. (2008) who attribute flares in a some AGN to the interaction of a travelling shock with a stationary recollimation shock at the position of the core. However, in these circumstances, where the features concerned are at best marginally resolved by VLBI arrays, key signatures of the shocks, such as the ratio of the upstream to downstream 
brightness, cannot be measured directly or even inferred (as in the case of the more extended structure in 3C 120). For this reason, the best way to confirm the existence of recollimation shocks is to identify their characteristic features, among which the polarization signature is one of the most promising candidates.

In the past, comparison between model and observed polarization has been difficult since, at the resolution of available data, the core represents a blend of many different components and is often dominated by newly emerging knots rather than the quiescent features that might be associated with the conical shocks. Recently, however, the availability of high quality images from the Very Long Baseline Array (VLBA) at frequency $43 \mathrm{GHz}$ has provided a new source of data in which to search for radio cores that might consist of simple, quiescent structures. Despite the availability of such images for a number of years, the polarization is often not displayed optimally for this purpose. Visualizations in which the polarized intensity is represented by the length of polarization rods often give a poor impression of its distribution in marginally resolved structures - and are not ideal for comparison with models. Contour maps of polarized flux density with polarization rods superimposed are far better in this respect, but rarely seem to appear in published work.

This paper presents images of the quasar $1803+784$ drawn from the $7 \mathrm{~mm}$ VLBA polarization survey by Jorstad et al. (2005, 2007), the polarization contour maps being shown here for the first time. They reveal a distinctive polarization structure that is discussed in terms of the conical shock models.

\section{Observations}

The compact, flat-spectrum radio source $1803+784$ is associated with a BL Lac object at redshift $z=0.6797$ (Lawrence et al., 1996). The structure of the radio source takes the 
form of a one-sided and apparently curved jet. A series of bimonthly VLBI observations at $43 \mathrm{GHz}$ revealed three superluminal components with apparent speeds in the range $\simeq 10-16 c($ Jorstad et al., 2005). Observations at lower frequencies with less frequent sampling suggested that most prominent features in the jet were stationary, though one superluminal component with apparent speed $\simeq 19 c$ was detected (Britzen et al. 2010).

The observations used in this paper were obtained as part of a larger program of investigation in which a sample of AGN was observed at roughly 2 month intervals over the course of approximately 3 years, from 1998 to 2001. The sources were observed by the VLBA at frequency $43 \mathrm{GHz}$ and by a number of other telescopes operating in the mm, sub-mm and optical bands. The full results were presented by Jorstad et al. (2005) and Jorstad et al. (2007).

Inspection of images of $1803+784$ presented in Jorstad et al. (2005) reveals radial patterns of polarization rods, reminiscent of results from the conical shock modelling of Cawthorne (2006). To facilitate a closer comparison, the polarized intensity contour images, in which the polarization angles are represented by rods of constant length, were generated, and two of these are shown in Fig. 1, where total intensity contour images and fractional polarization gray-scale images are also shown. The images have been rotated so that the apparent jet axes are vertical. The direction of North is shown in the total and polarized intensity plots.

In this paper, Faraday rotation is assumed to be unimportant at $43 \mathrm{GHz}$ in $1803+784$. This is implied by the approximate reflection symmetry of the polarization rods about the jet axis, as seen in Fig. 1; it is unlikely that such symmetry would survive a significant amount of Faraday rotation. Furthermore, Mahmud et al. (2009) measured Faraday rotation associated with core region, using images at $43 \mathrm{GHz}$ and lower frequencies. The largest RM detected was $1200 \mathrm{rad} \mathrm{m}^{-2}$, corresponding to a rotation of only $3.5^{\circ}$ at $43 \mathrm{GHz}$. 

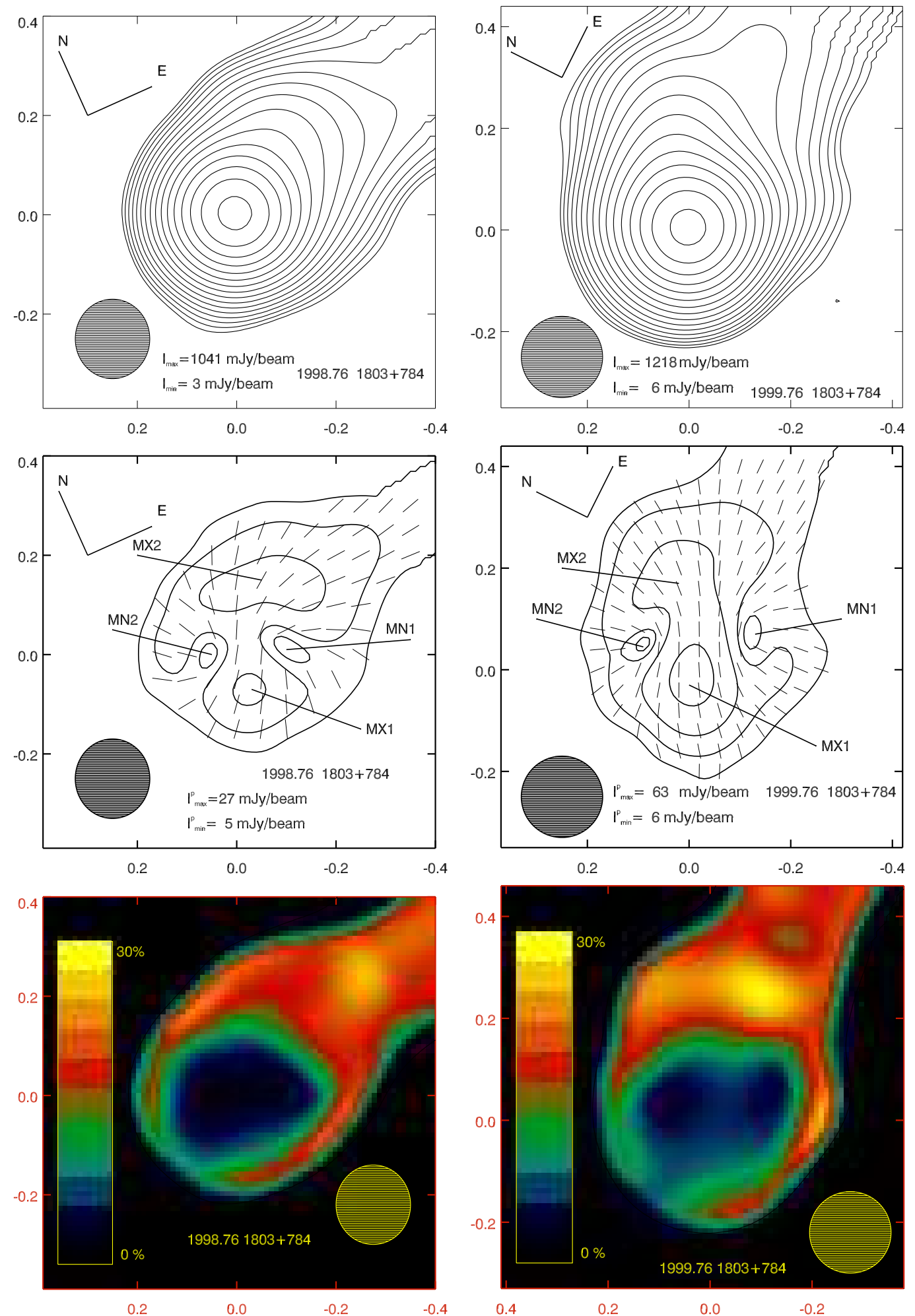

Fig. 1. - Images of $1803+784$ at $43 \mathrm{GHz}$ in total intensity, polarized intensity and polarization $E$-field rods of constant length, and fractional polarization. The total intensity contours increment by a factor of $\sqrt{2}$ and the polarized intensity contours increment by a factor of 2 . The RA and Dec scale is in mas. The polarization minima and maxima are labelled MN1 
The most striking aspect of these images is the unusual and complex polarized intensity structure. As expected, many of the images do show a radial pattern of polarization rods similar to that found in some of the conical shock-simulations shown by Cawthorne (2006). However there are also features that the conical shock models do not reproduce. The polarized intensity is often double-peaked. These two peaks, which define an approximate symmetry axis, are of comparable brightness, the North-Western of the two (i.e. the furthermost downstream) being weaker at some epochs and generally less compact. On a line orthogonal to the symmetry axis between the two peaks, there are two minima of polarization, one either side of the axis. This type of structure is particularly evident at the epochs October 1998 and October 1999 shown in Fig. 1 but is present to some extent at most of the seventeen observations that were made. For clarity, the maxima and minima of polarized intensity are marked on the corresponding plots in Fig. 1.

This paper addresses the question of whether such structures could arise from the conical shock model or one of its variants.

\section{Models}

Any model for the structures shown in Fig. 1 must account for the two minima in polarized intensity, and must therefore entail cancellation between orthogonal components of polarization. In addition, the model must explain the two on-axis components of polarized intensity and pattern of polarization rods that radiate from a position between them. The required cancellation of polarization can result in a number of ways. For example, if the jet contained a partially ordered magnetic field, then such an effect might result from superposition of emission from toroidal and poloidal field components; such models are discussed, e.g., in Canvin and Laing (2004). However, the radial pattern of polarization rods is reminiscent of the conical shock models of Cawthorne \& Cobb (1990) and Cawthorne 
(2006). These form an attractive basis for this investigation, since, as discussed in Section 1, they are a component of recollimation shocks, which are often identified with the high frequency cores in AGN (e.g., Marscher et al., 2008). In this model the polarization minima result from superposition of polarized emission from the collimating and decollimating shocks: in the region between the polarized maxima, their polarization angles rotate in opposite directions with increasing distance from the axis, making this type of cancellation a very probable occurrence.

\subsection{Conical shock models.}

The models used in this paper are essentially those of Cawthorne (2006), based on a framework presented in Lind \& Blandford (1985). The shocks are modelled as conical surfaces, having semi-opening angle $\eta$, that are coaxial with the jet. The axis of the system is inclined at an angle $\theta$ to the line of sight, where $\theta$ and $\eta$ are measured in the reference frame of the shock (which is assumed to be at rest with respect to the AGN). In the region upstream of shock the flow is assumed to be parallel to the jet axis with speed $c \beta_{u}$. As a result of the first shock, the flow is deflected through an angle $\xi$ away from the axis and decelerated to speed $c \beta_{d}$. The system is shown in Fig. 2.

In previous work (Cawthorne \& Cobb 1990, Cawthorne 2006, Agudo et al. 2012) simulated polarization images were formed by making the following assumptions: the emission is dominated by one of the two conical shock waves (or, in the case of Agudo et al. 2012, only one such shock occurs); the shock structures are assumed to be steady over timescales less than or comparable to a light crossing time; the source is assumed to be

optically thin for frequencies at which the emission is to be simulated; the radiating material is visible only near the shock surface, i.e., it cools rapidly; and the upstream densities of radiating particles and magnetic energy are uniform across the jet's cross section. Both up 
and downstream plasmas are assumed to have a relativistic equation of state and sound speed $c / \sqrt{3}$.

Among models of this type, those of greatest interest here have jet axes making small angles to the line of sight and largely disordered upstream magnetic fields (corresponding to $f=0$ in Cawthorne, 2006). It is these models that reproduce the roughly radial pattern of $E$-field polarization rods found in the core of $1803+784$.

An example is shown in Fig. 3. Here, the emission is computed for a single conical shock front where the jet axis is inclined at angle $\theta=1.5^{\circ}$ to the line of sight, the upstream Lorentz factor is $\gamma_{1}=10$ and the cone angle is $\eta=6^{\circ}$. With these values, the downstream Lorentz factor is $\gamma_{2}=7.8$, and the flow is deflected toward the shock surface by $2.1^{\circ}$.

The first and second panels show model total and polarized intensities, respectively. The third panel shows variation of fractional polarization along the axis. The fourth, fifth and sixth panels show the effects of convolution with a beam: the fourth panel shows total intensity contours with polarization rods of length proportional to polarized intensity and the restoring beam (FWHM) in the top right; the fifth panel shows polarized intensity contours with polarization rods of constant length; the sixth panel shows a gray-scale plot of fractional polarization.

Even when the jet axis makes such a small angle to the line of sight, the intensity patterns are still quite strongly asymmetric, in the sense that the total intensity and polarization are higher on the downstream (upper) than on the upstream (lower) side of the shock. In total intensity, this arises because the angle between the downstream velocity and the line of sight is much smaller, and therefore the Doppler factor is much larger, on the upper than on the lower part of the cone surface. The same effect also boosts the polarized intensity, but the main reason for the polarization asymmetry is that, in the rest frame of the downstream flow, the angle between the line of sight and the normal to shock surface 
is much closer to $90^{\circ}$ on the upper than on the lower part of the cone surface. Hence, the magnetic field appears much more highly ordered on the upper shock surface, and the fractional polarization is therefore much higher there than on the lower side. This is clearly seen in panel 3 of Fig. 3.

Note that in these simulations, the shock can be regarded either as decollimating (in which case the flow is downward in the figure and the conical surface is opening toward the observer) or collimating (when the flow is upward and the conical surface opens away from the observer) (see also Fig. 2 in Cawthorne, 2006).

While the polarization distribution does reproduce approximately a radial pattern of polarization, it reproduces neither the double peak in polarized intensity seen on the axis of the core in $1803+784$, nor the polarization minima either side of it. If the viewing angle is increased, the polarization becomes rapidly more strongly asymmetric, leaving just a simple fan of polarization rods associated with the upper part of the shock. As the viewing angle is reduced further, so the entire polarization structure rapidly becomes circularly symmetric. It seems that, with a purely disordered upstream magnetic field, there is no combination of parameters that will reproduce the polarization distributions shown in Fig. 1. At this point, therefore, an extension of the conical shock model is considered.

\subsection{Recollimation shocks.}

In numerical simulations and laboratory experiments, conical shocks occur in pairs. The first, collimating shock over-compensates for the initial divergence of the flow, and this is corrected by the second, decollimating shock. The two shocks are coaxial and meet at

their apexes, or possibly at a small Mach disc. In this paper, such structures are represented by the model shown in Fig. 4. The polarization distribution from such a system would be 
the sum of two distributions, each similar to that of Fig. 3, panel 5, one being reflected about the horizontal axis of the plot. This clearly offers the possibility of a structure with two peaks in polarized flux density on the jet axis. Slightly less obviously, it also raises the possibility of pairs of polarization minima along a line perpendicular to the axis. These minima result from cancellation due to the almost orthogonal polarizations contributed by the convolved images of the two shock structures. It therefore seems worth exploring whether the observed polarization could result from this type of structure.

Using the analytical approach, the polarization of synchrotron radiation from such structures can only be determined by introducing some additional assumptions, above and beyond those used by Cawthorne (2006), most of which relate to the behavior of the plasma between the two shocks; they are:

1. The initial and final fluid velocities are essentially parallel to the jet axis. In fact, numerical simulations show that in both regions, the flow is likely to be parallel to the axis near the axis, but diverging away from the axis at larger radii (J.L. Gomez, private communication). This would be difficult to combine with the approach used here, where the requirement that the shock front is a perfect cone demands that the flow velocities (within any half-cross section) are parallel.

2. The flow is diverted toward the axis by the first, collimating shock, and then realigned with the axis by the second (decollimating) shock. The cone angle of the second shock is determined by the need to realign the flow. (The effects of the converging upstream flow at the second shock can be taken into account using the approach of Nalewajko, 2009).

3. It is assumed that, downstream from the first shock, the plasma is made turbulent, such that following its passage through the emitting layer, the magnetic field returns to a disordered state. The high radio frequency core polarization in AGN is generally 
low and also highly variable both in intensity and position angle, a fact that is best explained if the upstream magnetic field is largely turbulent (e.g. Hughes, 2005). This is also true of the optical polarization, and the correlation between the polarization angles in the two frequency bands suggests the emission arises in the same region (Gabuzda \& Sitko 1994, D’Arcangelo et al., 2007, D'Arcangelo, 2010). In the case of 0420-014, D'Arcangelo et al. (2007) estimated that the emitting region consisted of some 600 cells, so the scale of the cells is likely to be much smaller than the size of the emitting region. Shocks are likely to cause a turbulent downstream flow if the gas pressure exceeds the magnetic pressure (an implicit assumption here, since the effect of the field on the shock dynamics has been ignored) (e.g., Hughes \& Eilek, 1990). If the downstream flow is turbulent on a similar length scale the to the upstream flow, then the imprint of the shock on the magnetic field structure could be lost quite quickly, even if the turbulent velocity component is significantly less than the flow velocity. Observationally, there is relatively little evidence to show how far downstream the ordered field survives, due to the difficulty of imaging interknot emission.

4. As in Cawthorne (2006), the emitting plasma is assumed to cool rapidly. Here, it is further assumed that the rest-frame plasma emissivity returns rapidly to its upstream value, and that emitting layers at the two shock fronts are of equal width. The most likely cooling process is synchrotron radiation, though inverse Compton scattering of synchrotron radiation and of high frequency (optical or infra-red) radiation from an accretion torus may also contribute. The validity of this assumption is discussed at the end of this section. Note, however, that if the electrons do age rapidly through radiative cooling, and if the core component could be isolated from its neighbouring features, spectral ageing should be manifest in a steepening of the spectrum at frequencies above $40 \mathrm{GHz}$. This could be an important test of the model when suitable 
spectra are available.

5. Between the two shocks, the flow is converging and the plasma is subject to a two-dimensional, radial compression. During this process, it is assumed that the disordered state of the magnetic field is maintained by turbulence.

6. This compression causes an enhancement of the magnetic field and the particle density, potentially by a large factor. If the re-tangling of the field does not increase the magnetic flux density (as it may well, e.g. Scheuer, 1987), then compression of the jet radius by a factor $\left(r / r_{0}\right)$ may result in an enhancement in synchrotron emissivity by a factor as great as $\left(r / r_{0}\right)^{-q}$ where $q=4 \frac{2}{3}$ or $q=6$ for spectral index $\alpha=0.5$ or 1, respectively (Leahy, 1991). Such a strong dependence presents a problem for the model proposed here, as it results in images that are completely dominated by the second shock, disallowing the formation of polarized features such as double peaks on axis and polarization minima. The effect of finite synchrotron lifetime may mitigate the effect of compression. However, the root of the problem is that the semi-analytical model of Fig. 4 almost certainly produces too great a convergence of the flow between the two shocks, as it neglects any response of the flow to increasing internal pressure. This view is supported by the MHD simulations produced by Roca-Sogorb et al. (2008) and Roca-Sogorb (2011) which clearly indicate recollimation shocks in which the largest width of the decollimating shock is not much smaller than that of the collimating shock. This is clearly a failing of the semi-analytic model. As a result of this, and in the absence of any obvious analytical way to allow for effects that might moderate the sensitivity of the plasma emissivity, slightly weaker (and ad hoc) forms of dependence have been investigated. It was found that an emissivity proportional to $\left(r / r_{0}\right)^{-n}$ where $n \simeq 3$ allowed some interaction between the polarizations of the two shocks, and this dependence has been adopted for the purpose of the present 
work. This ad hoc solution is, of course, not entirely satisfactory, but it provides a convenient way to proceed, pending a fully-numerical investigation.

These assumptions are required to connect the states of the fluid at the two shock fronts. They allow this investigation to determine whether the idea of using twin recollimation shocks to explain the core polarization of $1803+784$ is a plausible one. If the results are promising, then the behavior of the plasma between the shocks needs to be investigated using a numerical approach.

The geometry of the shock structure is summarized in Fig. 4. This model is characterized by three input parameters, namely, the upstream Lorentz factor $\gamma_{1}$, the cone angle of the first (decollimating) shock, $\eta_{1}$, and the viewing angle $\theta$. That said, there is an approximate equivalence of simulated images for which $1 / \gamma_{1}, \eta_{1}$, and $\theta$ are scaled in proportion; it is thus the two parameters $\theta / \gamma_{1}^{-1}$ and $\eta / \gamma_{1}^{-1}$ that determine the results. In addition, for any value of $\gamma_{1}$, the range of values of $\eta$ is constrained fairly strongly: $\eta$ must exceed the minimum value for the formation of a shock front, given by $\sin \left(\eta_{\min }\right)=1 /(\sqrt{2} \gamma \beta)$; however, values much larger than this result in large deflections, strong compression of the plasma before it reaches the second shock, and significant reductions in the Lorentz factor of the flow, none of which is helpful in explaining the structure of $1803+784$. Furthermore, the value of the viewing angle, $\theta$, is constrained to

Table 1: Comparison between data and model parameters for October 1998 observations.

\begin{tabular}{llllll}
\hline \hline & $\Delta r_{\text {max }} / b_{\text {maj }}$ & $\Delta r_{\text {min }} / b_{\text {maj }}$ & $p_{\text {max }} / p_{\text {min }}$ & $m_{\text {core }}$ & $m_{\text {jet }}$ \\
\hline data & $1.38 \pm 0.13$ & $1.00 \pm 0.088$ & $1.06 \pm 0.18$ & $0.032 \pm 0.004$ & $0.084 \pm 0.008$ \\
model & 1.05 & 0.97 & 1.09 & 0.038 & 0.049 \\
data - model $\mid / \sigma$ & 2.6 & 0.4 & 0.2 & 1.6 & 4.4 \\
\hline
\end{tabular}




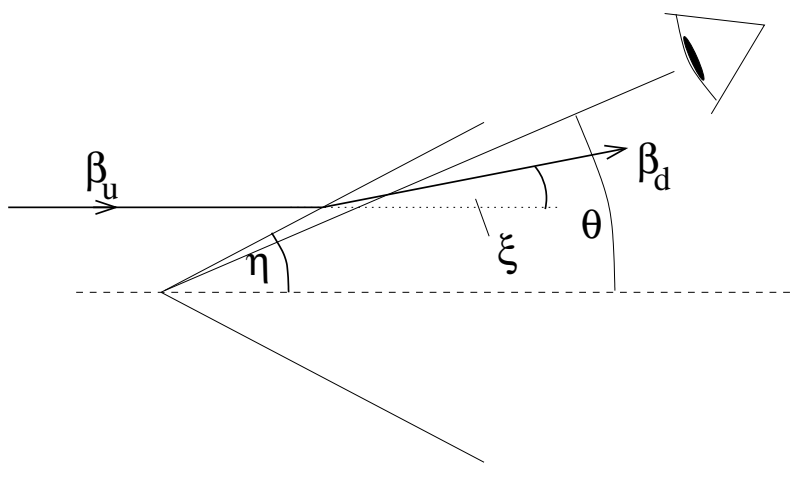

Fig. 2.- Illustration of conical model. The flow is initially parallel to the jet axis, but is deflected by the shock through an angle $\xi$.

Table 2: Comparison between data and model parameters for October 1999 observations.

\begin{tabular}{llllll}
\hline \hline & $\Delta r_{\text {max }} / b_{\text {maj }}$ & $\Delta r_{\text {min }} / b_{\text {maj }}$ & $p_{\text {max }} / p_{\text {min }}$ & $m_{\text {core }}$ & $m_{\text {jet }}$ \\
\hline data & $1.25 \pm 0.25$ & $1.30 \pm 0.13$ & $0.48 \pm 0.09$ & $0.05 \pm 0.005$ & $0.15 \pm 0.03$ \\
model & 1.06 & 1.11 & 0.72 & 0.06 & 0.04 \\
data - model $\mid / \sigma$ & 0.75 & 1.5 & 2.6 & 2.0 & 3.6 \\
\hline
\end{tabular}



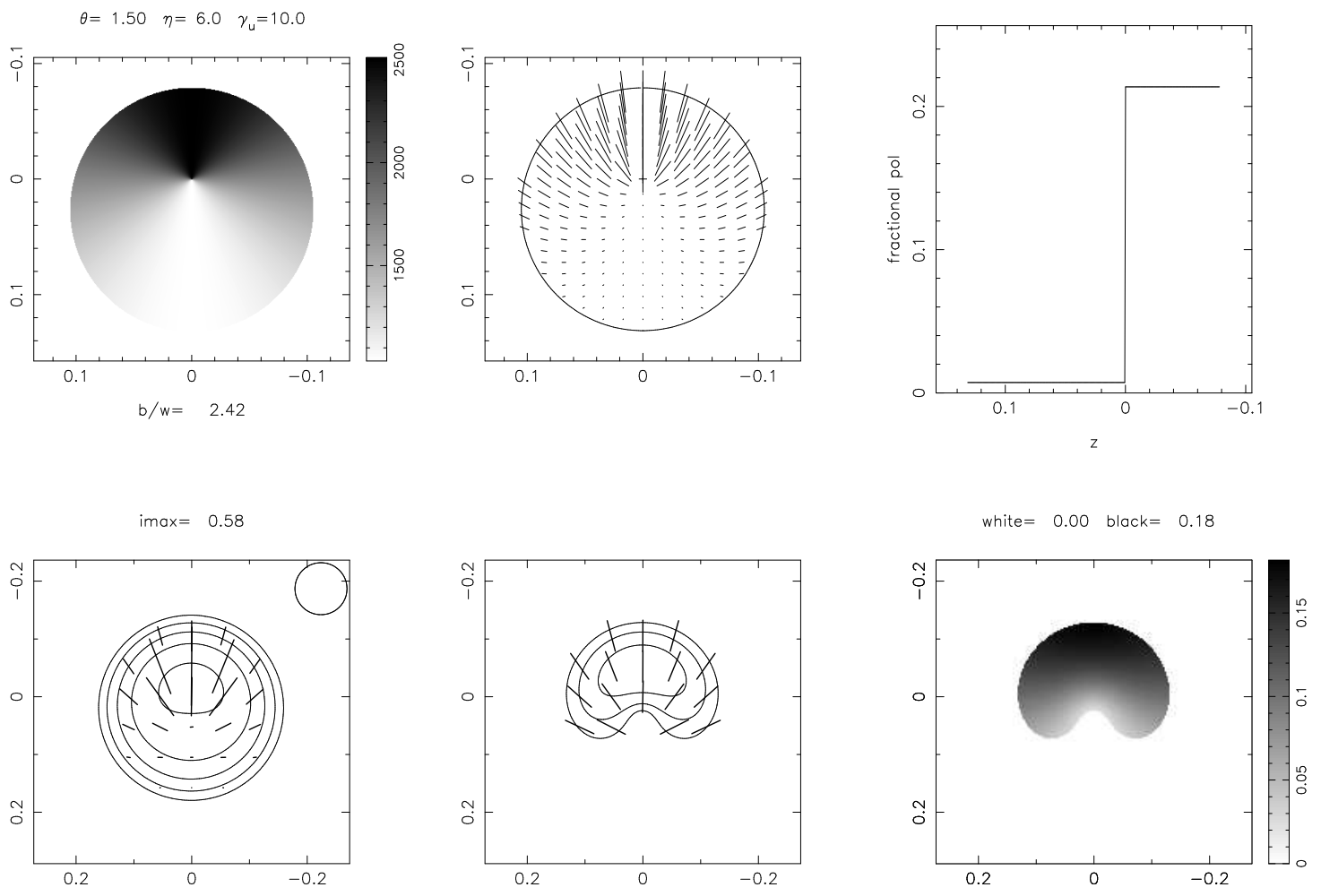

Fig. 3.- Illustration of emission from the single conical shock model with upstream Lorentz factor $\gamma_{u}=10$, cone semi-angle $\eta=6^{\circ}$, and an angle $\theta=1.5^{\circ}$ between the axis and line of sight. The first two panels show distributions of total intensity, $I$, and polarized intensity, $P$. The third panel shows the run of fractional polarization along the axis. The fourth and fifth panels illustrate the effects of convolution with the circular Gaussian beam shown in the top right of the fourth panel. They show, respectively, contours of $I$ and $P$, with polarization $E$ field rods proportional in length to $P$ (fourth panel) and constant in length (fifth panel). The value of $I_{\max }$ shown above the fourth panel represents the length of a polarization rod that corresponds to an intensity equal to the peak value of $I$. The lowest contours are $5 \%$ in $I$ and $15 \%$ in $P$, with contours stepping upward in factors of two. The sixth panel shows the distribution of fractional polarization post convolution, cut at the lowest $P$ contour. 
be less than the cone angle (to obtain the required radial pattern of polarization $E$ field). Hence the range of model parameters to be investigated is quite tightly restricted. As a result of the first shock the flow is deflected through an angle $\xi$ toward the axis and decelerated to speed $c \beta_{2}$. The second shock realigns the flow with the jet axis, further reducing the flow speed to $c \beta_{3}$.

The choice of Lorentz factor for $1803+784$ is not straightforward, as there are several superluminal components with a range of speeds (which themselves, vary with time) (Jorstad et al. 2005 and Britzen et al. 2010). Fortunately, as discussed above, this choice is not critical and for the present work a nominal upstream Lorentz factor $\gamma_{1}=10$ has been adopted. Doubling the Lorentz factor and halving the angles $\theta$ and $\eta$ (and the beam size) produces relatively minor changes in the simulated images.

The smallest possible choice of angle $\eta_{1}$, for the upstream shock, is $\eta_{\min } \simeq 4.1^{\circ}$, while for $\eta_{1}=7^{\circ}$, the jet Lorentz factor is halved on passage through the two shocks, and the deflection angle of $\simeq 3^{\circ}$ results in halving of the flow radius by the time the second shock is encountered. The results of greatest interest are therefore to be found between these two values of $\eta_{1}$.

In an attempt to characterize the degree of success of the simulations, several characteristic properties of the polarized intensity images were measured. These are: $\Delta r_{\text {max }} / b_{\text {maj }}$, the ratio of the separation of the polarization peaks on axis to the FWHM major axis of the beam, $b_{m a j} ; \Delta r_{m i n} / b_{m a j}$, the ratio of the separation of the polarization minima to $b_{m a j} ; p_{\text {core }} / p_{\text {jet }}$, the ratio of the flux densities of the two polarized peaks, and $m_{\text {core }}$ and $m_{\text {jet }}$, the fractional polarizations of these two features. (In this context, the upstream and downstream peaks in polarized intensity are labelled, respectively, as the 'core' and 'jet' components without implying any physical connotation.) The values of these quantities were obtained by plotting profiles of total and polarized intensity and fractional 
polarization along lines running along the axis, and perpendicular to the axis along a line plotted through the two minima. The uncertainties were also estimated from these plots, those in polarized flux density and fractional polarization being obtained as described by Hovatta et al. (2012). A $\chi^{2}$ parameter was defined for each of these quantities and the total $\chi^{2}$ was used to judge the level of agreement between model and data. A range of models was explored manually, by varying $\eta$ and $\theta$ in $0.25^{\circ}$ intervals. The size of the beam is, of course, unknown in model coordinates, For each pair of values $\eta, \theta$, the major axis was varied to find the value that minimized $\chi^{2}$. (The ratio $b_{m a j} / b_{m i n}$ and the beam position angle relative to the jet axis were held at the known values of 1.07 and $55^{\circ}$, respectively.) The comparison between the observed and model values of $\Delta r_{m a x} / b_{m a j}$ and $\Delta r_{m i n} / b_{m a j}$ provides a check for the self-consistency of this procedure.

It was found that, for $\gamma_{1}=10$ and values of $\eta_{1}$ in the region of greatest interest, $4.1^{\circ}<\eta_{1}<7^{\circ}$, the main features of the observations, i.e., the double peaks on axis and the double minima of the polarized intensity, were obtained for values of $\theta$ in the approximate range $1^{\circ}<\theta<2^{\circ}$. The values of the characteristic parameters and the deviations between model and observed values are shown in Tables 1 and 2 for the October 1998 and October 1999 images, respectively. For the October 1998 observations shown in Fig. 1, at $\eta_{1}=6^{\circ}$, the smallest $\chi^{2}$ occurs for $\theta=1.5^{\circ}$. The value of $\eta_{2}$ for this model is $4.95^{\circ}$ and the deflection angle $\xi=2.14^{\circ}$. The simulated images for this model are shown in Fig. 5 , in which the six panels are as described for Fig. 3. To facilitate comparison of the observed and simulated polarized intensity images, these are shown side by side in Fig. 6.

For the October 1999 observations, also shown in Fig. 1, a slightly smaller cone angle is favored. With $\eta_{1}=5^{\circ}, \eta_{2}=4.45^{\circ}$ and $\xi=1.14^{\circ}$, the smallest $\chi^{2}$ occurs for $\theta=1.75^{\circ}$. The simulated image for this model is similar to that shown in Fig. 5 (except that the 'jet' polarized intensity is less than that of the 'core') and so is not presented separately. 
Formally, the fits are not good. For both observations, the major source of discrepancy is the polarization of the outer 'jet' component, which has a much higher polarization than can be obtained in this model.

At this point, it is important to consider whether Assumption 4, that shocked gas cools quickly, is valid. The synchrotron lifetime of electrons in the shocked gas is

$$
\tau_{\text {synch }} \simeq 1.5\left(B / 5 \times 10^{-5} \mathrm{~T}\right)^{-3 / 2}\left(\nu_{\mathrm{obs}} / 43 \mathrm{GHz}\right)^{-1 / 2} \mathrm{yr}
$$

where the emission and observing frequencies are related by $\nu_{e m}=\nu_{o b s}(1+z) / D$, and a typical Doppler factor $D \simeq 13$ for the October 1998 model has been assumed. Given that, on submilliarcsecond scales, values several times $B \simeq 10^{-5} \mathrm{~T}$ are quite probable (e.g., O'Sullivan \& Gabuzda, 2009), synchrotron lifetimes may be as short as 1.5 years, resulting in a downstream flow distance $\gamma_{2} v_{2} \tau \simeq 10.5 \mathrm{ly}=3.2 \mathrm{pc}\left(\right.$ for $\left.\gamma_{2}=7\right)$. This compares to a deprojected separation of the two polarized maxima of $\simeq 60 \mathrm{pc}$ (for an inclination angle of $1.5^{\circ}$ ). Therefore, so long as the magnetic field is greater than about $10^{-5} \mathrm{~T}$, the emitting gas will cool significantly within a relatively short distance from the shock. At $B=5 \times 10^{-5}$, the observed turnover frequency will be approximately $10 \mathrm{GHz}$; the field should not be much larger than this value or the assumption that the optical depth is low breaks down.

\section{Discussion}

A full chi-squared analysis of the difference between the data and models presented has not been undertaken and is probably not appropriate: what such an analysis would show is that, in detail, the model and data fit rather poorly. This is a consequence of the many assumptions made in the very simple model, e.g. the cylindrical symmetry, which, the data shows, can only be approximate. Nevertheless, the previous section has shown that, for a range of plausible parameters, the recollimation shock model reproduces the principal 
features of the polarized intensity images in the core structure of $1803+784$. Furthermore, parameters can be found for which the model describes these features quantitatively: for most, model and data parameters agree to within 2.5 $\sigma$. Given the extreme simplicity of the model and the small number of free parameters, this appears to be an impressive level of agreement. The fractional polarization of the downstream (or 'jet') component, $m_{\text {jet }}$, is the property for which agreement is worst; its model polarization is too low by over $3.5 \sigma$. Allowing a larger value of $\eta_{1}$ does increase the fractional polarization of the 'jet' component, but this is at the expense of further depleting the kinetic energy of the downstream flow, and also increasing the fractional polarization (and therefore the contribution to $\chi^{2}$ ) for the 'core' component. The higher polarization associated with the 'jet' component may be due to some residual ordering of the magnetic field by the first shock that survives until the second shock is reached, and which is not taken into account by the model. This could lead to a higher degree of order in the magnetic field as the plasma emerges from the second shock and therefore a higher fractional polarization than the model allows. It is also possible that other effects not included in the model of Fig. 4, such as velocity shear, play a role ordering the magnetic field.

Fig. 5 shows that the convolved total intensity distribution of the model is clearly extended toward the lower, upstream side of the peak, as shown by the spacing of the contours. The reverse is true in the data, where an extension to the downstream side of the peak is a manifestation of the extended jet structure (which is not included in the model). The first panel of Fig. 5 shows clearly that the upstream extension in the model is due to the first of the two conical shocks, which is larger and fainter than the the second. The most obvious conclusion to draw from this is that the assumption of uniform flow between the two shocks has led to too great a degree of compression as the plasma passes between them. If the flow direction changes, deviating slightly away from the axis, the two conical shock waves would be more similar in projected size and emissivity, thereby largely eliminating 
the low brightness extension seen in the fourth panel of Fig 5 . As this emission is due to the parts of the shock structure that are farthest upstream (by quite a large margin, given the small cone angle), it may also be that this extended emission is suppressed by opacity.

A further source of disagreement is the precise orientation of the polarization rods. There is a broad level of agreement between model and data: both have on-axis $E$ field parallel to the axis and a roughly radial pattern elsewhere. However, off axis, the polarization angle values do not agree precisely, and this is not reflected in the comparison between model and data presented in Section 3. The disagreement is clear from the orientation of the polarization rods in the outer parts of the source on the line joining the two polarization minima. In the data (Fig. 1) these are almost perpendicular to the axis, whereas in the model (Fig. 5, panel 5) they make an angle with the axis close to $60^{\circ}$. Inspection of the second panel in Fig. 5 shows that the polarization angles in the region farthest from the axis are determined largely by the first (and largest) of the two conical shocks in the region furthest upstream. This is the same region responsible for the unwanted upstream extension in total intensity and therefore the discrepancy may be also be reduced if the shocks are more equal in size, or if the upstream emission is suppressed by opacity, as suggested above.

From this discussion it is clear that, while the recollimation shock model seems very promising as a means of explaining the core polarization in $1803+784$, input from a numerical magnetohydrodynamical (MHD) investigation would be useful as a means to examine discrepancies between the model and the data.

In order to obtain a representation of the source, a very small viewing angle was required. At larger viewing angles, the double peaks on axis and the minima either side disappear, and the model produces a much smaller range of polarization angles than is seen in Figs. 1 and 5. Most of the sources in a sample of blazars will have viewing angles smaller 
than or comparable to $1 / \gamma$, where $\gamma$ is the Lorentz factor. In the model of Fig. 5 , the Lorentz factors are $\gamma_{2}=7.8$ and $\gamma_{3}=6.45$, respectively. Hence the emission is characterized by a Lorentz factor $\gamma \simeq 7$. For these Lorentz factors, the required polarization structures were only obtained for angles $\theta<2^{\circ} \simeq 0.035 \mathrm{rad}$. Hence it might be expected that only a fraction of order $(0.035 /(1 / 7))^{2} \simeq 0.06$ of sources in the sample would exhibit the interesting structure shown here. This is consistent with the fact that, of the 15 sources included in the sample of Jorstad et al. (2005), only one showed structure of this type. In more poorly aligned sources, fans of polarization rods covering a smaller range of polarization angles might be expected, and examples of this kind of structure were seen in the cores of the sources 0420-014 and 0528+134 which were also included in the Jorstad et al. (2005) sample.

If the interpretation presented here is correct, then it is interesting to compare the location of the recollimation shock, and hence of the radio core, with the core position in other sources. The core position has been estimated from component speeds and time lags between X-ray dips and the ejection of radio components in at least two radio jet sources: 3C 120 (Marscher et al., 2002) and 3C 111 (Chaterjee et al., 2011). In those sources the core location is between $10^{5}$ and $10^{6}$ times the black hole Schwarzchild radius, $R_{s}$. For $1803+784$, a BL Lac object, the black hole mass has been estimated to be in the region of $4 \times 10^{8} M_{\odot}$, (Wang et al., 2004), though the scaling relation from which this is estimated involves a significant scatter (at least half a decade). The black hole-core separation must be at least the height of one of the conical shocks. The projected height can be estimated roughly as about half the separation of the polarization peaks in Fig. 1, i.e. about 0.1 mas. If the line of sight angle $\theta \simeq 1.5^{\circ}$ (as in the model of Table 1 ) the deprojected height is then about $30 \mathrm{pc}$. These values give a black hole-core separation of approximately $3 \times 10^{6} R_{s}$, somewhat larger than for 3C 120 or 3C 111 . However, $\theta$ could be larger if the Lorentz factors are correspondingly smaller, and the black hole mass is likely to be uncertain by 
at least a factor of three. Given these uncertainties, it is at least possible that the black hole-core separation in units of $R_{s}$ is of the same order as that in the other sources.

A further feature evident from Fig. 1 is the variation in direction of the symmetry axis between the two epochs shown. In fact, there is a definite wobble in the direction of the axis from epoch to epoch, similar to that noted by Denn et al. (2000) and Stirling et al. (2003) in BL Lac. These variations are too large to be caused by possible changes in orientation of the nearly circular beam. What is noteworthy here is how much more evident are these variations in polarization for this distinctive type of structure than in total intensity. A detailed analysis of these variations is left for future work.

\section{Conclusions.}

Observations of a number of sources from the AGN sample studied by Jorstad et al. (2005, 2007) at $43 \mathrm{GHz}$ revealed unusual patterns of polarization structure reminiscent of structures predicted by the Conical Shock models of Cawthorne \& Cobb, (1990) and Cawthorne (2006). The most interesting of these was found in the source $1803+784$, which, at most epochs, has two on-axis polarization peaks and two polarization minima along a line perpendicular to the axis and lying between the two peaks. Such features were visible in this source at most of the 17 observing epochs. Single conical shocks alone acting on plasma with a largely disordered upstream magnetic field cannot reproduce these properties, but the analytical simulations presented in this paper show that twin conical shocks of the kind that occur in recollimation shocks can yield these characteristics for a range of reasonable model parameters, when the jet axis and the observer's line of sight are well aligned. The agreement between model and data is described by defining five parameters that characterize the polarization image. For four of these, the agreement is reasonable, but the fifth, the fractional polarization associated with downstream polarized (or 'jet') 
component, is discrepant by $4.4 \sigma$ (October 1998) and 3.6 $\sigma$ (October 1999). Some aspects of the total intensity and polarization angle distributions are also in disagreement. The root of these disagreements seems to be the simplifications that were made regarding the progress of the emitting gas between the two conical shocks, and this points to the need for a numerical (MHD) investigation in which such assumptions are not required.

Despite these disagreements, the ability of such a simple model with a small number of free parameters to reproduce the principal polarization features observed in $1803+784$ seems impressive, and suggests that the core polarization in $1803+784$ may well be associated with a recollimation shock. This would appear to be the most direct evidence so far for associating the core emission in an extragalactic radio source with a structure of this kind. It strengthens the view that the core components in high frequency observations represent the point at which the quiescent jet structure is first disturbed, and that this disturbance may in many instances be due to recollimation shock structure.

\section{Acknowledgments}

TVC thanks the Director of the Jeremiah Horrocks Institute at the University of Central Lancashire for a sabbatical that allowed completion of this work. He also thanks Dr. Denise Gabuzda and the Physics Department at University College Cork for providing sanctuary in which much of the work presented here was performed. Dr. Gabuzda also provided many useful comments on the paper, as did Dr. Jose-Luis Gómez and the anonymous referee. Mr. Colm Coughlan assisted with some of the analysis presented here. The research at Boston University was supported in part by US National Science Foundation grant AST-0907893. The VLBA is an instrument of the National Radio Astronomy Observatory. The National Radio Astronomy Observatory is a facility of the National Science Foundation operated under cooperative agreement by Associated 
Universities, Inc.

\section{REFERENCES.}

Agudo, I., Gómez, J.-L., Martí, J.-M, Ibáñez, J.-M., Marscher, A.P., Alberdi, A., Aloy, M.-A., Hardee, P. E. 2001, ApJ, 549, L183

Agudo, I. 2009, ASPC, 402, 300

Agudo, I., Gómez, J.-L., Casadio, C., Cawthorne, T. V., Roca-Sogorb, M. 2012, ApJ, 752, 92

Britzen, S., Kudryavtseva, N. A., Witzel, A., Campbell, R. M., Ros, E., Karouzos, M.,

Mehta, A., Aller, M.F., Aller, H.D., Beckert, T., Zensus, J. A. 2010, A\&A, 511, 57

Canvin, J. R., Laing, R. A. 2004, MNRAS, 350, 1342

Cawthorne, T. V., Cobb, W. K. 1990, ApJ, 350, 536

Cawthorne, T. V. 2006, MNRAS, 367, 851

Chatterjee, R., Marscher, A.P., Jorstad, S. G., Markowitz, A., Rivers, E., Rothschild, R.E., McHardy, I. M., Aller, M. F., Aller, H. D., Lahteenmaki, A., Tornikson, M., Harrison, B., Agudo, I., Gómez, J.-L., Taylor, B. W., Gurwell, M. 2011, ApJ, 743, 43

Daly, R. A., Marscher, A.P. 1988, ApJ, 334, 539

D’Arcangelo, F. D., Marscher, A. P., Jorstad, S. G., Smith, P. S., Larionov, V. M.,

Hagen-Thorn, V. A., Kopatskaya, E. N., Williams, G. G., Gear, W. K. 2007, ApJ, 659, L107

D’Arcangelo, F. D. 2010, Ph.D. Thesis, Boston Univ.

Denn, G., Mutel, R., Marscher, A.P. 2000, ApJS, 129, 61

Eilek, J. A., Hughes, P. A. 1991, Beams and Jets in Astrophysics, (Cambridge: CUP), 428

Falle, S. A.E. G., Wilson, M. J. 1985, MNRAS, 216, 79

Gabuzda, D. C., Sitko, M. L. 1994, AJ, 107, 884

Gómez, J.-L., Martí, J. M., Marscher, A.P., Ibáñez, J. M., Marcaide, J. M. 1995, ApJ, 449, L19 
Gómez, J.-L., Martí, J. M., Marscher, A. P., Ibáñez, J. M., Alberdi, A. 1997, ApJ, 482, L33 Jorstad, S. G., Marscher, A.P., Lister, M. L., Stirling, A. M., Cawthorne, T. V., Gear, W. K., Gómez, J.-L., Stevens, J. A., Smith, P. S., Forster, J. R., Robson, E. I. 2005, AJ, 130, 1418

Jorstad, S. G., Marscher, A.P., Stevens, J. A., Smith, P.S., Forster, J.R., Gear, W.K., Cawthorne, T. V., Lister, M. L., Stirling, A. M., Gómez, J.-L., Greaves, J. S., Robson, E. I. 2007, AJ, 134, 799

Hovatta, T., Lister, M., Aller, M. F., Aller, H. D., Homan, D. C., Kovalev, Y. Y., Pushkarev, A. B., Savolainen, T. 2012, AJ, 144, 105

Hughes, P. A. 2005, ApJ, 621, 635

Lawrence, C. R., Zucker, J. R., Readhead, A. C.S., Unwin, S. C., Pearson, T. J., Xu W. 1996, ApJS, 107, 541

Leahy, J. P. 1991, in Beams and Jets in Astrophysics, Ed. P. A. Hughes, CUP, p. 100

Lind, K. R., Blandford, R. D. 1985, ApJ, 295, 358

Mahmud, M., Gabuzda, D. C., Bezrukovs, V. 2009, MNRAS, 400, 2

Marscher, A.P., Jorstad, S. G., Gómez, J.-L., Aller, M. F., Terasantra, H., Lister, M., Stirling, A. 2002, Nature, 417, 625

Marscher, A.P., Jorstad, S. G., D’Arcangelo, F. D., Smith, P.S., Williams, G. G., Larionov, V.M., Oh, H., Olmstead, A. R., Aller, M. F., Aller, H. D. and 13 coathors 2008, Nature, 212,2002

Nalewajko, K. 2009, MNRAS, 395, 524

O’Sullivan, S. P., Gabuzda, D. C. 2009, MNRAS, 400, 26

Papageorgiou, A., Cawthorne, T. V., Stirling, A. M., Gabuzda, D. C., Polatidis, A. G. 2006, MNRAS, 373, 449

Roca-Sogorb, M., Perucho, M., Gómez, J.-L., Marti, J. M., Anton, L., Aloy, M. A., Agudo, I. 2008, in Extragalactic Jets: Theory and Observation from Radio to Gamma Ray, ASP 
Conference Series, 386, 488

Roca-Sogorb, M. 2011, PhD Thesis, University of Valencia

Roca-Sogorb, M., Gómez, J.-L., Agudo, I., Marscher, A. P., Jorstad, S. G. 2010, ApJ, 712, 160

Scheuer, P. A. G. 1987, in Astrophysical Jets and their Engines, Ed. W. Kundt, D. Reidel, p. 137

Stirling, A. M., Cawthorne, T. V., Stevens, J. A., Jorstad, S. G., Marscher, A. P., Lister, M. L., Gómez, J.-L., Smith, P. S., Agudo, I., Gabuzda, D. C., Robson, E. I., Gear, W. K. MNRAS, 341, 405

Wang, J.-M., Luo, B., Ho, L. C. 2004, ApJ, 615, L9 


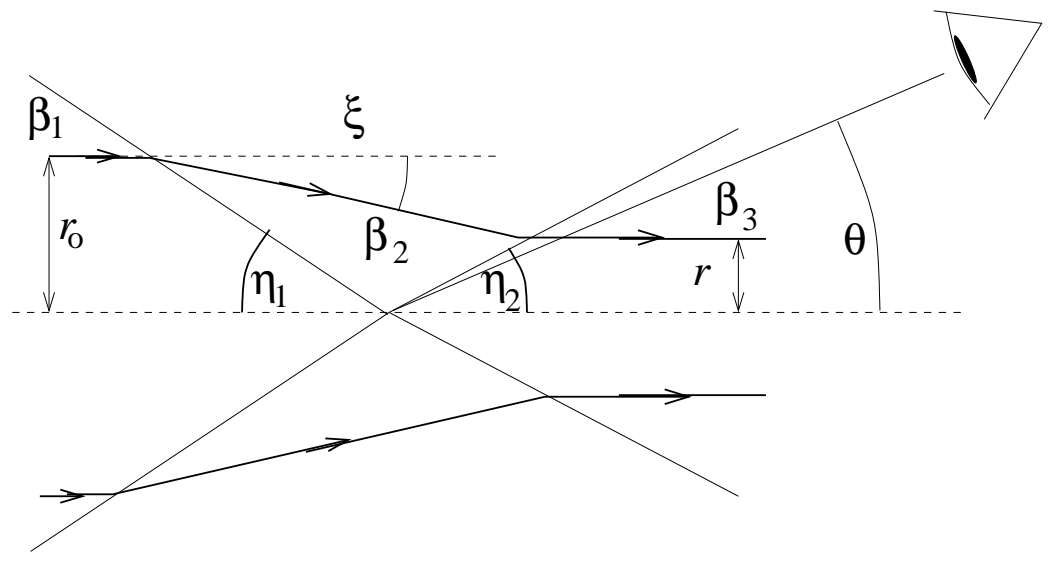

Fig. 4. - Illustration of recollimation shock model. The flow is initially parallel to the jet axis, but is deflected by the first shock through an angle $\xi$ then realigned with the axis by the second shock. 
$\theta=1.50^{\circ} \eta_{1}=6.00^{\circ} \eta_{2}=4.95^{\circ} \gamma_{1}=10.0$
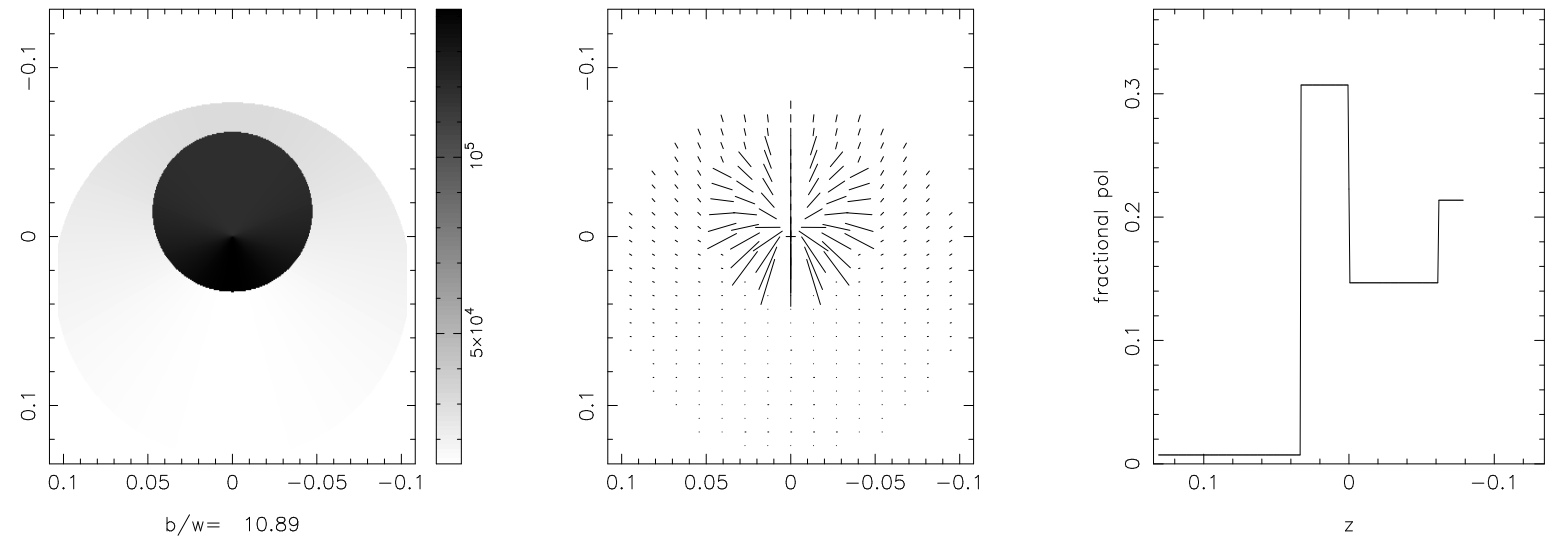

$\operatorname{imax}=1.87$
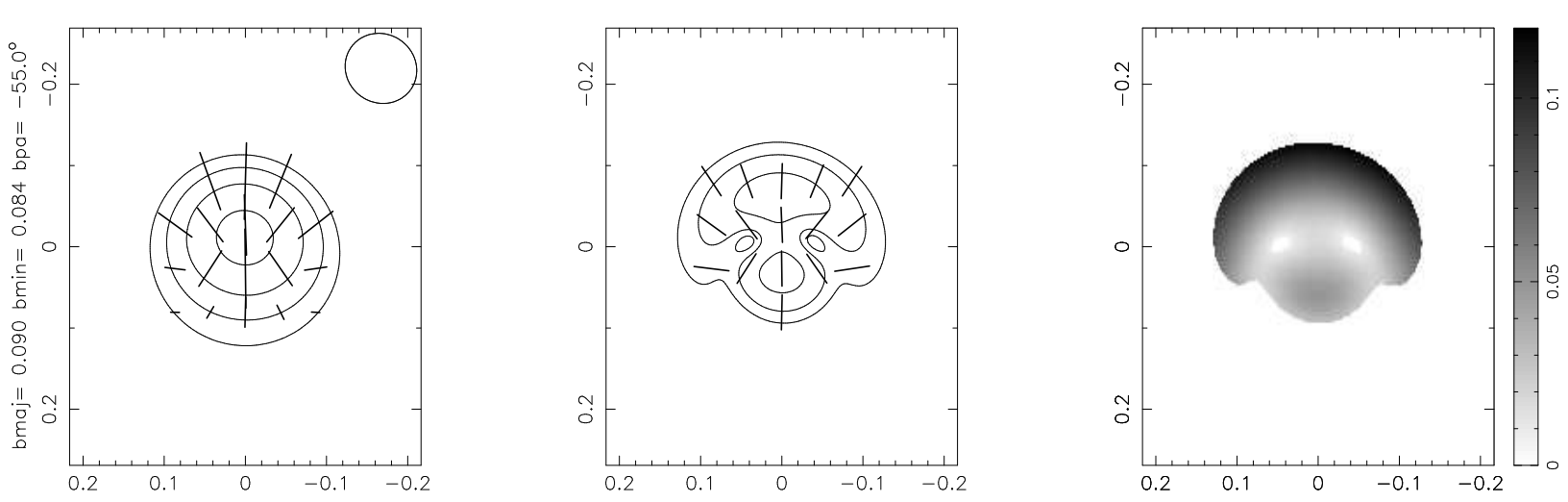

Fig. 5.- Images for the double shock model corresponding to parameter values shown above the first panel. The panels are as described in Fig. 3. The lowest contours in the convolved total and polarized intensity contour maps are 10 and 18 percent of their respective peak values. 

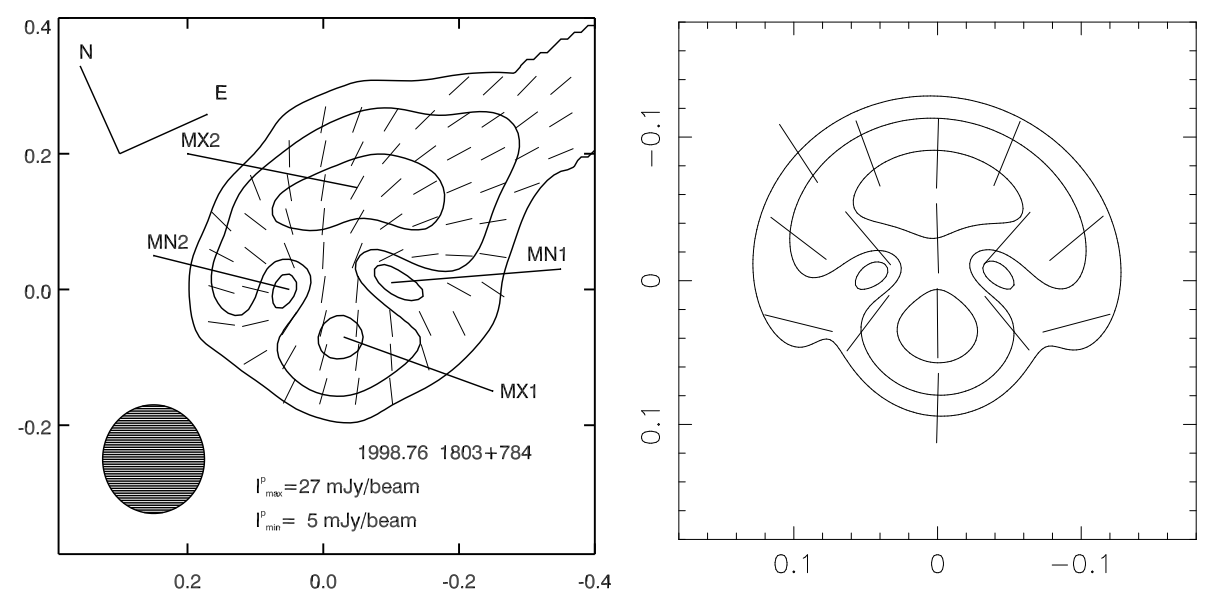

Fig. 6.- The October 1998 polarized intensity image from Fig. 1 is shown alongside the simulated polarized intensity image from Fig. 5, to facilitate comparison of the two. 